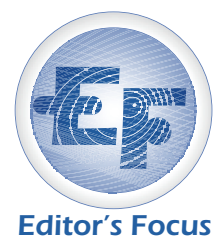

\title{
Intrinsic charm production of doubly charmed baryons: Collider vs. fixed-target
}

\author{
Ramona $\operatorname{Vogt}^{1,2^{*}}$, and Stanley J. Brodsky ${ }^{2 *}$ \\ ${ }^{1}$ Nuclear and Chemical Sciences Division, Lawrence Livermore National Laboratory, Livermore, CA 94551, USA; \\ ${ }^{2}$ Department of Physics, University of California, Davis, CA 95616, USA; \\ ${ }^{3}$ Stanford Linear Accelerator Center, Stanford University, Stanford, CA 94309, USA
}

Received November 22, 2019; accepted December 17, 2019; published online December 24, 2019

Citation: R. Vogt, and S. J. Brodsky, Intrinsic charm production of doubly charmed baryons: Collider vs. fixed-target, Sci. China-Phys. Mech. Astron. 63, 221066 (2020), https://doi.org/10.1007/s11433-019-1496-7

The LHCb Collaboration has measured the doubly charmed baryon, $\Xi_{c c}^{++}(u c c)$, through two different decay channels $[1,2]$ in $p+p$ collisions at center-of-mass energies of 7, 8 and 13 $\mathrm{TeV}$. While they have performed similar searches for the doubly charmed baryon $\Xi_{c c}^{+}(d c c)$, it has not yet been observed by LHCb [3]. However, the fixed-target experiment, SELEX, at Fermilab, reported the observation of the $\Xi_{c c}^{+}$in two different decay channels with a $600 \mathrm{GeV}$ charged hyperon beam $[4,5]$. The beam was composed of an admixture of $\pi^{ \pm}, p, \bar{p}$ components as well as the $\Sigma^{-}$hyperon. Much discussion has arisen over the fact that the fixed-target observations have not been easily reproduced by the collider experiments at higher energies, where one might have expected the production rates to be much higher due to the orders of magnitude increase in the center of mass energy with LHCb.

As we explain, the dramatic discrepancy between $\mathrm{LHCb}$ and SELEX could be due to the different kinematic domains of the two experiments. In fact, we show that if the $\Xi_{c c}^{+}$ and $\Xi_{c c}^{++}$are produced within the framework of the intrinsic heavy-quark model $[6,7]$, the production kinematics in the fixed-target experiment, SELEX, are more favorable for detection of double charm baryons than LHCb, even with their forward-focused detector. See also ref. [8].

Intrinsic heavy quark Fock states, an intriguing aspect of

*Corresponding authors (Ramona Vogt, email: rlvogt@lbl.gov; Stanley J. Brodsky, email: sjbth@slac.stanford.edu) hadron light-front wavefunctions in QCD, stand in marked contrast with standard production cross sections based on gluon splitting, where only a small fraction of the incident gluon momentum goes to the production of heavy hadrons. These intrinsic heavy quark Fock states are remarkably efficient at producing heavy hadrons at maximal rates and minimal energy, as we now describe.

Consider a heavy-quark loop ( $Q \bar{Q}$ loop) insertion to the proton self-energy. If the $Q \bar{Q}$ loop is attached to just one valence quark by gluons, cutting such a diagram yields the standard gluon splitting contribution to the proton structure function and the heavy quarks appear at very small proton momentum fractions $x$. However, if the heavy quark loop is, instead, attached to two or more valence quarks in the proton self-energy, cutting such a diagram leads to the "intrinsic" heavy-quark contribution to the proton light-front wavefunction. In quantum chromodynamics, the probability for such an intrinsic heavy $Q \bar{Q}$ pair in the proton scales as $1 / M_{Q}^{2}$, in contrast to heavy $\ell \bar{\ell}$ lepton pairs in QED where the probability for heavy lepton pairs to exist in an atomic wavefunction scales as $1 / M_{\ell}^{4}$. This difference in mass scaling distinguishes Abelian from non-Abelian theories.

This mass scaling also suggests that light-front wavefunctions decrease strongly with the invariant mass of the Fock state. The rapid decrease of the light-front wavefunction with the invariant mass squared, $\mathcal{M}^{2}=\left(\sum_{i} k_{i}^{\mu}\right)^{2}=$ 
$\sum_{i}\left(k_{\perp i}^{2}+m_{i}^{2}\right) / x_{i}$, of the Fock state constituents in colorconfining AdS/QCD models [9], implies that the probability for such states is maximized when the constituents have equal rapidity and the heavy quarks carry the greatest light-front momentum.

Heavy quarks thus carry a significant fraction of the momentum in an intrinsic heavy quark Fock state. For example, the charm quark in a $|u u d c \bar{c}\rangle$ state carries about $25 \%$ of the proton momentum: $x_{c} \sim 0.25$ [10]. The comoving constituents come on shell after a collision and coalesce into final state charm hadrons, such as $\Lambda_{c}$ with $x_{\Lambda_{c}} \sim x_{c}+x_{u}+x_{d} \sim 0.58$ [10]. When the number of quarks in a Fock state increases, the average $x$ of all quarks in the state decreases. Thus, in a $|u u d c \bar{c} c \bar{c}\rangle$ state, $x_{c} \sim 0.16$ and $x_{\Xi_{c c}^{++++}}=x_{c}+x_{c}+x_{q} \sim 0.43$, see Table 1 for the average $x$ values for $\Xi_{c c}^{+}$, $\Xi_{c c}^{++}$and their charge conjugate states from relevant meson and baryon projectiles for SELEX and LHCb, along with the minimum number of particles required in the Fock state to produce double charm baryons by coalescence. For a summary of evidence for intrinsic charm production, see ref. [7].

In a fixed-target configuration, the average $x$ values for the double charm baryons shown in Table 1, while forward

Table 1 Table of average $x$ values for $\Xi_{c c}^{+}, \Xi_{c c}^{++}$and their charge conjugates in the minimal Fock state configuration required for production by coalescence with $\pi^{-}, \pi^{+}, p$ and $\Sigma^{-}$projectiles

\begin{tabular}{|c|c|c|c|c|c|c|c|c|}
\hline & \multicolumn{2}{|c|}{$\pi^{-}(\bar{u} d)$} & \multicolumn{2}{|c|}{$\pi^{+}(u \bar{d})$} & \multicolumn{2}{|c|}{$p($ uud $)$} & \multicolumn{2}{|c|}{$\Sigma^{-}(d d s)$} \\
\hline & $n_{h}$ & $\langle x\rangle$ & $n_{h}$ & $\langle x\rangle$ & $n_{h}$ & $\langle x\rangle$ & $n_{h}$ & $\langle x\rangle$ \\
\hline$\Xi_{c c}^{+}(d c c)$ & 6 & 0.5 & 8 & 0.389 & 7 & 0.434 & 7 & 0.436 \\
\hline$\Xi_{c c}^{++}(u c c)$ & 8 & 0.389 & 6 & 0.5 & 7 & 0.434 & 9 & 0.323 \\
\hline$\overline{\Xi_{c c}^{+}}(\bar{d} \overline{c c})$ & 8 & 0.389 & 6 & 0.5 & 9 & 0.324 & 9 & 0.323 \\
\hline$\overline{\Xi_{c c}^{++}}(\overline{u c c})$ & 6 & 0.5 & 8 & 0.389 & 9 & 0.324 & 9 & 0.323 \\
\hline
\end{tabular}

compared to central production as in typical pQCD calculations where one of the initiating partons comes from the projectile and the other from the target, are all within the acceptance of a forward detector. In this case also the average $x$ is equivalent to the Feynman $x, x_{\mathrm{F}}$, of the measured hadron. With a predominantly $\Sigma^{-}$component of the beam, it is more probable to produce a $\Xi_{c c}^{+}$than a $\Xi_{c c}^{++}$with SELEX. The $x_{\mathrm{F}}$ distributions for the final state $\Xi_{c c}^{+}$and $\Xi_{c c}^{++}$and their charge conjugates are shown in Figure 1(a). To compare to the rapidity range covered by $\mathrm{LHCb}$, in the center of mass frame of the collision, with $\sqrt{s} \sim 35 \mathrm{GeV}$, the rapidity of the double charm baryons is $y_{E_{c c}} \sim 1.8$, well within the SELEX kinematic domain. The high $x_{\mathrm{F}}$ domain is also accessible at the proposed AFTER fixed-target experiment [11] at the LHC, with $\sqrt{s}=115 \mathrm{GeV}$, a more natural regime to test the SELEX results.

With LHCb, the kinematics are effectively identical for the $\Xi_{c c}^{+}$and $\Xi_{c c}^{++}$with a proton beam. However, because the proton has two up quarks and only one down, $\Xi_{c c}^{++}$production is twice as probable as $\Xi_{c c}^{+}$. If one considers the charge conjugates, the two doubly charmed baryons are equally probable. Their overall rate is decreased because an extra light $q \bar{q}$ pair must also exist in the Fock state. While the LHCb detector has the most forward acceptance of all the spectrometers at the LHC, $2<\eta<5$, in the collider configuration, the $\mathrm{LHCb}$ acceptance would measure only a small fraction of doubly charmed baryons produced by intrinsic charm since $x_{\mathrm{F}}=\left(2 m_{T} / \sqrt{s}\right) \sinh y$. Thus a particle carrying $43.4 \%$ of the beam momentum, would have an average rapidity of $y \sim 6.56$ at $7 \mathrm{TeV}$, falling mostly outside the LHCb acceptance, see Figure 1(b). Only the lower $x$ tails of the distributions are in the measurable range. To quantify this, approximately $2 \%$

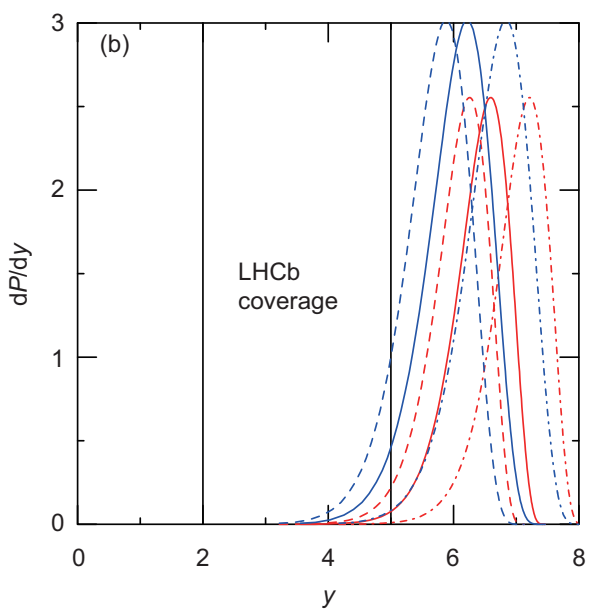

Figure 1 (Color online) (a) The unit-normalized probability distributions for $\Xi_{c c}^{+,++}$and $\overline{\Xi_{c c}^{+,++}}$production from $\pi^{-}$(solid blue), proton (solid red), $\Sigma^{-}$(dashed red), and $\pi^{+}$(dashed blue) are shown as a function of momentum fraction $x$. The distributions from a 9-particle Fock configuration from $p$ and $\Sigma^{-}$are given by the dot-dashed red curve. See Table 1 for which final states correspond to each distribution. (b) The unit-normalized probability distributions for $\Xi_{c c}^{++++}$ production from a 7-particle proton Fock state (red curves) and for $\overline{\Xi_{c c}^{+,++}}$production from a 9-particle proton Fock state. Since the rapidity range depends on center-of-mass energy, the distributions are shown for 5 (dashed), 7 (solid) and 13 (dot-dashed) TeV. The coverage of the LHCb detector, $2<y<5$, is also shown. 
of the charge conjugate probability distribution is within the LHCb acceptance while, for the $\Xi_{c c}^{+}$and $\Xi_{c c}^{++}$states themselves, the percentage of the probability distribution within the acceptance drops to $\sim 0.3 \%$. At the maximum $\mathrm{LHCb}$ energy, $\sqrt{s}=13 \mathrm{TeV}$, the acceptance from intrinsic charm states falls nearly to zero. Thus the higher the center-of-mass energy, the greater the boost to large rapidity and the less likely it becomes for these particles to be detected. Indeed, the lower $x$ of the charge-conjugate states would make them more likely to be detected by LHCb even though the probability of their production is reduced.

Other discrepancies between the fixed-target and collider results still remain. It would be natural to expect to observe both isospin partners $\Xi(c c u)^{++}$and $\Xi(c c d)^{+}$at similar masses at $\mathrm{LHCb}$ modulo the factor of two due to up and down quark numbers in the proton. However, the mass of the $\Xi_{c c}^{++}$reported by SELEX is $\sim 100 \mathrm{MeV}$ away from that reported by $\mathrm{LHCb}$. The lifetime reported by SELEX is also nearly an order of magnitude shorter. These experimental discrepancies need to be resolved. In addition, if SELEX observed the double charm baryon, the radiative decay of $\Xi(c c d)^{+}[3620]$ to $\Xi(c c d)^{+}[3519]+\gamma(100 \mathrm{MeV})$ should have been seen by LHCb. However as shown in ref. [8], this radiative decay could be dynamically and kinematically suppressed and therefore be comparable to the weak decay.
This work of R. V. was performed under the auspices of the U.S. Department of Energy by Lawrence Livermore National Laboratory (Grant No. DE-AC52-07NA27344) and supported by the U.S. Department of Energy, Office of Science, Office of Nuclear Physics (Nuclear Theory) (Grant No. DE-SC-0004014). The research of S. J. B. was supported by the Department of Energy contract (Grant Nos. DE-AC02-76SF00515, and OSTI ID: 1576972).

1 R. Aaij, et al. (LHCb Collaboration), Phys. Rev. Lett. 119, (2017).

2 R. Aaij, et al. (LHCb Collaboration), Phys. Rev. Lett. 121, (2018).

3 R. Aaij, et al. (LHCb Collaboration), Sci. China-Phys. Mech. Astron. 63, 221062 (2020), arXiv: 1909.12273 hep-ex.

4 M. Mattson, et al. (SELEX Collaboration), Phys. Rev. Lett. 89, 112001 (2002).

5 A. Ocherashvili, et al. (SELEX Collaboration), Phys. Lett. B 628, 18 (2005).

6 S. J. Brodsky, P. Hoyer, C. Peterson, and N. Sakai, Phys. Lett. B 93, 451 (1980); S. J. Brodsky, C. Peterson, and N. Sakai, Phys. Rev. D 23, 2745 (1981).

7 S. J. Brodsky, A. Kusina, F. Lyonnet, I. Schienbein, H. Spiesberger, and R. Vogt, Adv. High Energy Phys. 2015, 1 (2015).

8 S. J. Brodsky, S. Groote, and S. Koshkarev, Eur. Phys. J. C 78, 483 (2018).

9 G. F. de Téramond, and S. J. Brodsky, Phys. Rev. Lett. 102, 081601 (2009).

10 T. Gutierrez, and R. Vogt, Nucl. Phys. B 539, 189 (1999).

11 J. P. Lansberg, V. Chambert, J. P. Didelez, B. Genolini, C. Hadjidakis, P. Rosier, R. Arnaldi, E. Scomparin, S. J. Brodsky, E. G. Ferreiro, F. Fleuret, A. Rakotozafindrabe, and U. I. Uggerhøj, Proc. Sci. 2012, 49 (2012), arXiv: 1207.3507. 